\title{
WILCZYNSKI'S AND FUBINI'S CANONICAL SYSTEMS OF DIFFERENTIAL EQUATIONS*
}

\section{BY E. P. LANE}

1. Introduction. The projective differential geometry of surfaces has been studied extensively in the United States by Wilczynski, Green, and others, using the invariants and covariants of a completely integrable system of linear homogeneous partial differential equations. In Italy, much progress in projective differential geometry has been made by Fubini, Bompiani, and others, who have approached the subject from the point of view of differential forms and the absolute calculus. The normal coordinates of Fubini have been shown by him to be solutions of a canonical system of differential equations. It is the purpose of this note to show how Fubini's canonical differential equations may be obtained by Wilczynski's method, and to compare Wilczynski's and Fubini's canonical forms for the differential equations of a surface.

It is evidently desirable that geometers living on different sides of the Atlantic, writing in different languages, and using different analytic apparatus, but interested in the same subject, should be able to exchange ideas freely. It is hoped that this note will to some extent smooth the way for this commerce of ideas by showing how certain equations and formulas obtained in one notation may be written also in the other.

2. Analytic Foundation. Let the homogeneous coordinates $y^{(1)}, \cdots, y^{(4)}$ of a general point $P_{y}$ on a non-degenerate nondevelopable surface $S_{\boldsymbol{y}}$ be given as analytic functions of two independent variables $u, v$. If $S_{\nu}$ is referred to its asymptotic

* Presented to the Society, April 2, 1926. 
net, the four functions $y$ are solutions of a system of equations* of the form

$$
\left\{\begin{array}{l}
y_{u u}+2 a y_{u}+2 b y_{v}+c y=0, \\
y_{v v}+2 a^{\prime} y_{u}+2 b^{\prime} y_{v}+c^{\prime} y=0 .
\end{array}\right.
$$

The coefficients of these two equations are functions of $u, v$, and satisfy four integrability conditions, of which the only one that we shall use is

$$
a_{v}=b_{u}^{\prime}
$$

This condition shows that there exists a function $p$ such that

$$
p_{u}=a, \quad p_{v}=b^{\prime} .
$$

All transformations of the group

$$
y=\lambda(u, v) \bar{y}, \quad \bar{u}=U(u), \quad \bar{v}=V(v)
$$

are without geometric significance. In particular, the transformation $y=\lambda \bar{y}$ changes system (1) into another system of the same form whose coefficients are given by the following formulas:

$$
\left\{\begin{array}{l}
\bar{a}=a+\frac{\lambda_{u}}{\lambda}, \quad \bar{b}=b, \quad \bar{c}=\frac{1}{\lambda}\left(\lambda_{u u}+2 a \lambda_{u}+2 b \lambda_{v}+c \lambda\right), \\
\bar{a}^{\prime}=a^{\prime}, \quad \bar{b}^{\prime}=b^{\prime}+\frac{\lambda_{v}}{\lambda}, \quad \bar{c}^{\prime}=\frac{1}{\lambda}\left(\lambda_{v v}+2 a^{\prime} \lambda_{u}+2 b^{\prime} \lambda_{v}+c^{\prime} \lambda\right) .
\end{array}\right.
$$

3. Wilczynski's Canonical System. Wilczynski observes that we shall have $\bar{a}=\bar{b}^{\prime}=0$ if we choose $\lambda$ as a solution of the equations

$$
\frac{\lambda_{u}}{\lambda}+p_{u}=0, \quad \frac{\lambda_{v}}{\lambda}+p_{v}=0 .
$$

With this choice of $\lambda$ Wilczynski obtains for equations (1) the canonical form

$$
\left\{\begin{array}{l}
y_{u u}+2 b y_{v}+f y=0 \\
y_{v v}+2 a^{\prime} y_{u}+g y=0
\end{array}\right.
$$

* Wilczynski, First memoir, Transactions of this Society, vol. 8 (1907), pp. 233-260. 
wherein $f$ and $g$ are defined by the formulas

$$
f=c-a_{u}-a^{2}-2 b b^{\prime}, g=c^{\prime}-b_{v}^{\prime}-b^{\prime 2}-2 a a^{\prime} .
$$

The canonical system (W) is characterized by the conditions $a=b^{\prime}=0$. The largest subgroup of the group of transformations (4) that preserves the form of system (W) is

$$
y=\text { const. } \bar{y} / \sqrt{U^{\prime} V^{\prime}}, \quad \bar{u}=U(u), \quad \bar{v}=V(v) .
$$

If the semi-covariant points $y, y_{u}, y_{v}, y_{u v}$ are used as vertices of a local tetrahedron of reference with a unit point suitably chosen, the local coordinates of a point $x$ on $S_{y}$ near $P_{y}$ are represented by the series*

$$
\begin{aligned}
& x_{1}=1-\frac{1}{2} f \Delta u^{2}-\frac{1}{2} g \Delta v^{2}+\cdots, \\
& x_{2}=\Delta u-a^{\prime} \Delta v^{2}+\cdots, \\
& x_{3}=\Delta v-b \Delta u^{2}+\cdots, \\
& x_{4}=\Delta u \Delta v+\cdots .
\end{aligned}
$$

By means of these expansions the projective differential geometry of $S_{y}$ in the neighborhood of $P_{y}$ has been extensively studied.

4. Fubini's Canonical System. Referring to equations (5), let us observe that we shall have

$$
\frac{1}{2} \frac{\left(\bar{a}^{\prime} \bar{b}\right)_{u}}{\bar{a}^{\prime} \bar{b}}+\bar{a}=0, \quad \frac{1}{2} \frac{\left(\bar{a}^{\prime} \bar{b}\right)_{v}}{\bar{a}^{\prime} \bar{b}}+\bar{b}^{\prime}=0,
$$

if we choose for $\lambda$ a solution of the equations

$$
\text { (11) } \frac{\lambda_{u}}{\lambda}+p_{u}+\frac{1}{2} \frac{\left(a^{\prime} b\right)_{u}}{a^{\prime} b}=0, \frac{\lambda_{v}}{\lambda}+p_{v}+\frac{1}{2} \frac{\left(a^{\prime} b\right)_{v}}{a^{\prime} b}=0 \text {. }
$$

With this choice of $\lambda$ we obtain for equations (1) the canonical form

$$
\left\{\begin{array}{c}
y_{u u}-\frac{\left(a^{\prime} b\right)_{u}}{a^{\prime} b}-y_{u}+2 b y_{v}+\varphi y=0, \\
y_{v v}+2 a^{\prime} y_{u}-\frac{\left(a^{\prime} b\right)_{v}}{a^{\prime} b} y_{v}+\psi y=0,
\end{array}\right.
$$

* Wilczynski, Second memoir, Transactions of this Society, vol. 9 (1908), p. 98. 
wherein $\varphi$ and $\psi$ are defined by the formulas

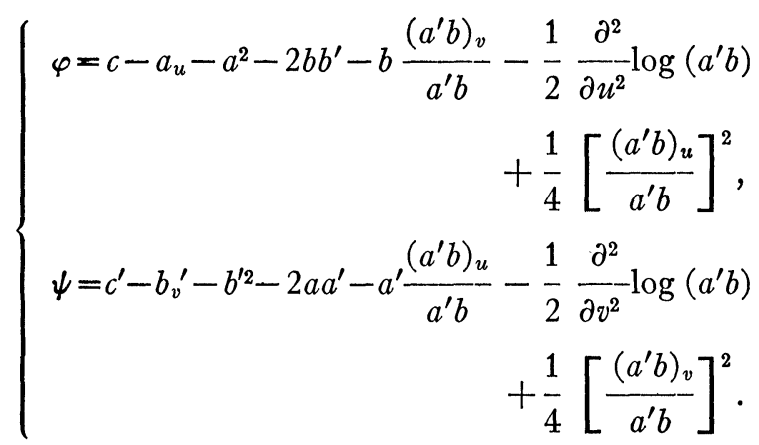

System (F) is equivalent to Fubini's canonical system. In fact Fubini shows* that his normal coordinates satisfy the equations

$$
y_{11}=\beta y_{2}+n y, \quad y_{22}=\gamma y_{1}+\nu y,
$$

wherein subscripts indicate covariant differentiation with respect to the quadratic form $2 \beta \gamma d u d v$. In light of the well known formulas for covariant differentiation, we see that these equations are precisely the same as equations $(F)$, provided that we place

$$
\beta=-2 b, \gamma=-2 a^{\prime}, n=-\varphi, \nu=-\psi .
$$

The canonical system $(\mathrm{F})$ is characteriaed by the conditions

$$
a=-\frac{1}{2} \frac{\left(a^{\prime} b\right)_{u}}{a^{\prime} b}, \quad b^{\prime}=-\frac{1}{2} \frac{\left(a^{\prime} b\right)_{v}}{a^{\prime} b} .
$$

The largest subgroup of the group of transformations (4) that preserves the form of system $(F)$ is

$$
y=c \bar{y}, \quad \bar{u}=U(u), \quad \bar{v}=V(v), \quad(c=\text { const. }) .
$$

The effect of the most general transformation (15) on the coefficients of system $(\mathrm{F})$ is given by the equations

(16) $\bar{a}^{\prime}=\frac{U^{\prime}}{V^{\prime 2}} a^{\prime}, \quad \bar{b}=\frac{V^{\prime}}{U^{\prime 2}} b, \quad \bar{\varphi}=\frac{1}{U^{\prime 2}} \varphi, \quad \bar{\psi}=\frac{1}{V^{\prime 2}} \psi$.

* Fubini, Fondamenti di geometria proiettivo-differenziale di una superficia, Atti Der Lincei, (5), vol. 27 (1918), 2d semester, p. 45. 
Therefore $\varphi$ and $\psi$, as well as $a^{\prime}$ and $b$, are invariants. Moreover, it is evident that under this transformation the points $y_{u}, y_{v}, y_{u v}$ are covariant. A geometric description of these points and an interpretation of the invariants $\varphi$ and $\psi$ will be furnished in Section 6.

5. Transformation from System (W) to System (F). We shall now establish the transformation between the local tetrahedron with vertices at the points $y, y_{u}, y_{v}, y_{u v}$ for Wilczynski's canonical system (W) and the local tetrahedron with vertices at the points $y, y_{u}, y_{v}, y_{u v}$ for Fubini's canonical system $(\mathrm{F})$.

In equations (9) let us replace $f$ and $g$ by the expressions given in equations (7). Then, for convenience, let us multiply each of the series thus obtained by a common proportionality factor, namely, the power series for the function $Y$ satisfying equations (6),

$$
\begin{aligned}
1-a \Delta u-b^{\prime} \Delta v+\frac{1}{2}\left(a^{2}-a_{u}\right) & \Delta u^{2}+\left(a b^{\prime}-a_{v}\right) \Delta u \Delta v \\
& +\frac{1}{2}\left(b^{\prime 2}-b^{\prime}{ }_{v}\right) \Delta v^{2}+\cdots .
\end{aligned}
$$

In this way we obtain the expansions

$$
\left\{\begin{aligned}
x_{1}= & 1-a \Delta u-b^{\prime} \Delta v+\left(a^{2}+b b^{\prime}-\frac{1}{2} c\right) \Delta u^{2}+\left(a b^{\prime}-a_{v} \Delta u \Delta v\right) \\
& +\left(b^{\prime 2}+a a^{\prime}-\frac{1}{2} c^{\prime}\right) \Delta v^{2}+\cdots \\
x_{2}= & \Delta u-a \Delta u^{2}-b^{\prime} \Delta u \Delta v-a^{\prime} \Delta v^{2}+\cdots \\
x_{3}= & \Delta v-b \Delta u^{2}-a \Delta u \Delta v-b^{\prime} \Delta v^{2}+\cdots \\
x_{4}= & \Delta u \Delta v+\cdots
\end{aligned}\right.
$$

These series represent the coordinates of a point $x$ on $S_{y}$ near $P_{y}$, in the notation of system (1), the tetrahedron of reference being the semi-covariant tetrahedron whose vertices are the points $y, \rho, \sigma, z$; the semi-covarients $\rho, \sigma, z$ being defined* by the equations

$$
\rho=y_{u}+a x, \sigma=y_{v}+b^{\prime} x, z=y_{u v}+b^{\prime} y_{u}+a y_{v}+\left(a b^{\prime}+a_{v}\right) y .
$$

If a point has coordinates $x_{1}, \cdots, x_{4}$ referred to the tetrahedron $y, \rho, \sigma, z$ and has coordinates $\bar{x}_{1}, \cdots, \bar{x}_{4}$ referred to the tetrahedron $y, y_{u}, y_{v}, y_{u v}$ for system (1), then we have

$$
x_{1}+x_{2} \rho+x_{3} \sigma+x_{4} z=k\left(\bar{x}_{1} y+\bar{x}_{2} y_{u}+\bar{x}_{3} y_{v}+\bar{x}_{4} y_{u v}\right) \text {, }
$$

\footnotetext{
* Wilczynski, First memoir, p. 248.
} 
where $k$ is a proportionality factor. Thus we obtain the following equations of transformation between these two tetrahedrons :

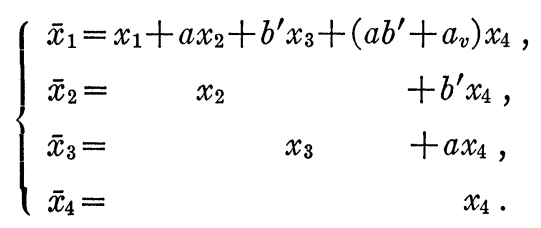

Using equations (17) and (18) we obtain then expansions for the coordinates of a point $x$ on $S_{y}$ near $P_{y}$, in the notation of system (1), the tetrahedron of reference being the tetrahedron $y, y_{u}, y_{v}, y_{u v}$ for system (1):

$$
\left\{\begin{array}{lr}
\bar{x}_{1}=1-\frac{1}{2} c \Delta u^{2}-\frac{1}{2} c^{\prime} \Delta v^{2}+\cdots, \\
\bar{x}_{2}= & \Delta u-a \Delta u^{2}-a^{\prime} \Delta v^{2}+\cdots, \\
\bar{x}_{3}= & \Delta v-b \Delta u^{2}-b^{\prime} \Delta v^{2}+\cdots \\
\bar{x}_{4}= & \Delta u \Delta v+\cdots
\end{array} .\right.
$$

If in equations (19) we substitute for $a$ and $b^{\prime}$ the expressions given by equations (14) and replace $c, c^{\prime}$ by $\varphi, \psi$ respectively, we obtain the coordinates of a point $x$ on $S_{y}$ near $P_{y}$ in the notation of system $(\mathrm{F})$, the tetrahedron of reference being the tetrahedron $y, y_{u}, y_{v}, y_{u v}$ for system (F). Equations (13) enable us to write the same coordinates in the notation actually used by Fubini.

Therefore it is possible to pass from an equation in local coordinates based on system (W) to the corresponding equations based on system (F) by using in succession equations (7), (18), (14), and substituting $\varphi$ for $c, \psi$ for $c^{\prime}$.

6. Geometric Applications. In the notation of system (W) the equation of the quadric of Lie, or osculating* quadric, is

$$
x_{1} x_{4}-x_{2} x_{3}+2 a^{\prime} b x_{4}^{2}=0 .
$$

By the method of the preceding section the equation of this

* Wilczynski, Second memoir, p. 82. 
quadric in the notation of system (F) is found to be

(20) $x_{1} x_{4}-x_{2} x_{3}+\left[2 a^{\prime} b+\frac{1}{2} \frac{\partial^{2}}{\partial u \partial v} \log \left(a^{\prime} b\right)\right] x_{4}^{2}=0$.

Fubini, using system (F), has remarked* that the line joining the two points $y$ and $y_{u v}$ is the projective normal. Green, using system $(W)$, has called $†$ the line joining the points $y$ and $z$, where

$$
z=y_{u v}+\frac{1}{2} \frac{\left(a^{\prime} b\right)_{v}}{a^{\prime} b} y_{u}+\frac{1}{2} \frac{\left(a^{\prime} b\right)_{v}}{a^{\prime} b} y_{v}
$$

the pseudo-normal. These two lines are easily shown to be the same by means of the transformation established in the preceding section. In fact, the local coordinates of $P_{z}$ referred to the tetrahedron $y, y_{u}, y_{v}, y_{u v}$ for system (1) are found by equations (18) to be

$$
\text { ( ( ) } \left., \frac{1}{2} \frac{\left(a^{\prime} b\right)_{v}}{a^{\prime} b}+b^{\prime}, \frac{1}{2} \frac{\left(a^{\prime} b\right)_{u}}{a^{\prime} b}+a, 1\right) \text {. }
$$

Equations (14) show that, for system (F), this point is on the line joining the points $y$ and $y_{u v}$.

We are now in position to describe geometrically the points $y_{u}, y_{v}, y_{u v}$ for system (F). A simple calculation based on equation (20) shows that the reciprocal polar of the projective normal, with respect to the quadric of Lie, crosses the tangent of the asymptotic $v=$ const. at the point $y_{u}$ and crosses the tangent of the asymptotic $u=$ const. at the point $y_{v}$. Thus these points are characterized. In order to characterize the point $y_{u v}$, we observe that the locus of the point $y_{u}$, as $u, v$ vary, is a surface covariant to $S_{y}$. The tangent at the point $y_{u}$ to the curve $u=$ const. on the surface $y_{u}$ intersects the projective normal at the point $y_{u v}$, as does also the tangent at the point $y_{v}$ to the curve $v=$ const. on the surface $y_{v}$.

* Fubini, Alcuni risultati di geometria proiettivo-differenziale, ATtI DEI Lincei, (5), vol. 32 (1923), 2d semester, p. 324.

$\dagger$ Green, Memoir on the general theory of surfaces, Transactions of This Society, vol. 20 (1919), p. 126. 
Inspection of system $(\mathrm{F})$ reveals that the tangent at the point $y_{u}$ to the curve $v=$ const. on the surface $y_{u}$ is the line $y_{u} y_{v}$ if, and only if, $\varphi=0$. Similarly, the tangent at the point $y_{v}$ to the curve $u=$ const. on the surface $y_{v}$ is the line $y_{u} y_{v}$ if, and only if, $\psi=0$.

We shall now determine the developables and focal surfaces of the congruence of lines $y_{u} y_{v}$ reciprocal to the congruence of projective normals. Any point $Y$ on a line $y_{u} y_{v}$ (except the point $y_{v}$ ) is given by an expression of the form

$$
Y=y_{u}+\lambda y_{v} \text {. }
$$

As $P_{y}$ moves along a curve $v=v(u)$ on $S_{y}, P_{y}$ describes a curve. A point on the tangent of this curve is given by

$Y^{\prime}=Y_{u}+Y_{v} \frac{d v}{d u}=\left(\lambda+\frac{d v}{d u}\right) y_{u v}+\left(\phi+\lambda \frac{d v}{d u} \psi\right) y+() y_{u}+\left(\right.$ ) $y_{v}$.

If $P_{y}$ is a focal point of the line $y_{u} y_{v}$ and if the curve on $S_{y}$ corresponds to a developable of the congruence of these lines, then we have

$$
\lambda+\frac{d v}{d u}=0, \quad \varphi+\lambda \frac{d v}{d u} \psi=0 .
$$

Eliminating $\lambda$, we obtain the differential equation of the developables of the congruence,

$$
\varphi d u^{2}-\psi d v^{2}=0
$$

And eliminating $d v / d u$, we obtain

$$
\psi \lambda^{2}-\varphi=0 \text {. }
$$

Solution of this quadratic determines the focal surfaces of the congruence. The significance of the simultaneous vanishing of $\varphi$ and $\psi$ is thus made evident.

7. Transformations from System (F) to System (W). If we have an equation in local coordinates based on system (F) and wish to find the corresponding equation based on the sytem (W) we may proceed as follows. Replacing $\varphi$ and $\psi$ by the expressions given for them in equations (12), we obtain the corresponding equation in the notation of system (1), the 
tetrahedron of reference being the covariant tetrahedron used with system $(\mathrm{F})$. The vertices of this tetrahedron, besides $P_{y}$, are the points $\rho, \sigma, z$ expressed in the notation of system (1) by the formulas

$$
\left\{\begin{array}{l}
\rho=y_{u}+(a-\beta) y, \quad \sigma=y_{v}+\left(b^{\prime}-\alpha\right) y, \\
z=y_{u v}+\left(b^{\prime}-\alpha\right) y_{u}+(a-\beta) y_{v}+\left[(a-\beta)\left(b^{\prime}-\alpha\right)+a_{v}-\beta_{v}\right] y .
\end{array}\right.
$$

wherein we have placed

$$
\alpha=-\frac{1}{2} \frac{\left(a^{\prime} b\right)_{v}}{a^{\prime} b}, \quad \beta=-\frac{1}{2} \frac{\left(a^{\prime} b\right)_{u}}{a^{\prime} b} .
$$

If a point has coordinates $x_{1}, \cdots, x_{4}$ referred to this tetrahedron $y, \rho, \sigma, z$ and has coordinates $\bar{x}_{1}, \cdots, \bar{x}_{4}$ referred to the tetrahedron $y, y_{u}, y_{v}, y_{u v}$ for system (1), we have the following equations of transformation:

$$
\left\{\begin{array}{lcr}
\bar{x}_{1}=x_{1}+(\alpha-\beta) x_{2}+\left(b^{\prime}-\alpha\right) & x_{3}+\left[(a-\beta)\left(b^{\prime}-\alpha\right)+a_{v}-\beta_{v}\right] x_{4} \\
\bar{x}_{2}= & x_{2} & +\left(b^{\prime}-\alpha\right) x_{4}, \\
\bar{x}_{3}= & x_{3} & +(a-\beta) x_{4} \\
\bar{x}_{4}= & & x_{4}
\end{array}\right.
$$

By means of this transformation we are able to find the equation of the given locus in the notation of system (1) and referred to the tetrahedron $y, y_{u}, y_{v}, y_{u v}$ for system (1). In order to obtain the desired equation, it only remains to place $a=b^{\prime}$ $=0$ and to replace $c, c^{\prime}$ by $f, g$ respectively.

University of Chicago 